\title{
Subcutaneous Trastuzumab for HER2-positive Breast Cancer - Evidence and Practical Experience in 7 German Centers
}

\author{
Trastuzumab s. c. beim HER2-positiven Mammakarzinom - Evidenz und \\ Erfahrungen aus der praktischen Anwendung in 7 deutschen Zentren
}

Authors

Affiliations
C. Jackisch ${ }^{1}$, V. Müller ${ }^{2}$, P. Dall ${ }^{3}$, R. Neumeister ${ }^{4}$, T.-W. Park-Simon ${ }^{5}$, A. Ruf-Dördelmann ${ }^{6}$, S. Seiler ${ }^{1}$, H. Tesch ${ }^{7}$, B. Ataseven ${ }^{8}$

The affiliations are listed at the end of the article.

Key words
trastuzumab
breast cancer
subcutaneous administration
Schlüsselwörter
Trastuzumab
Mammakarzinom
subkutane Therapie

Deutschsprachige Zusatzinformationen online abrufbar unter: www.thieme-connect.de/ ejournals/toc/gebfra

received $\quad 17.4 .2015$ revised 18.5.2015 accepted 18.5.2015

Bibliography

DOI http://dx.doi.org/ 10.1055/s-0035-1546172 Geburtsh Frauenheilk 2015; 75 : 566-573 @ Georg Thieme Verlag KG Stuttgart · New York . ISSN 0016-5751

\section{Correspondence}

Prof. Dr. Christian Jackisch

Sana Klinikum Offenbach

Obstetrics and Gynecology

Starkenburgring 66

63069 Offenbach

christian.jackisch@sana.de

\section{Abstract \\ $\nabla$}

A subcutaneous formulation of trastuzumab to treat patients with HER2-positive breast cancer is available since August 2013. The subcutaneous formulation is administered as a fixed dose of $600 \mathrm{mg}$ over a period of up to 5 minutes. The HannaH trial compared subcutaneous with intravenous administration and found comparable pharmacokinetics, efficacy and tolerability for both administration forms of trastuzumab in the neoadjuvant setting. The randomized crossover study PrefHer reported a clear preference from the patient's point of view for subcutaneous over intravenous administration of trastuzumab. The accompanying time-and-motion study reported a reduction concerning the total time spent for the institution as well as for the patient receiving trastuzumab s.c.. The experience of 7 German centers largely corresponded with the results of these studies. Patients expressed a clear preference for subcutaneous trastuzumab administration, with the time saved by the subcutaneous administration route cited as the greatest benefit. Although the existing reimbursement terms mean that centers will receive a lower remuneration, the centers' overall evaluation of the subcutaneous administration route for trastuzumab was overwhelmingly positive. The greatest benefit cited by the centers was the flexibility in scheduling patient appointments. This increased flexibility improved conditions in some centers which were experiencing pressures due to a shortage of staff, particularly at peak times. The general consensus, however, was that the remuneration systems for oncological treatments urgently need to be amended to ensure that the real costs of treatment are covered, even if the administration route has changed.

\section{Zusammenfassung \\ $\nabla$}

Seit August 2013 steht für Patientinnen mit HER2positivem Mammakarzinom die subkutane Applikationsform von Trastuzumab zur Verfügung, die in einer Fixdosis von $600 \mathrm{mg}$ über rund 5 Minuten verabreicht wird. In der HannaH-Studie wurden in der neoadjuvanten Therapie eine vergleichbare Pharmakokinetik, Wirksamkeit und Verträglichkeit beider Applikationsformen von Trastuzumab gezeigt. Die randomisierte Crossover-Studie PrefHer wies eine deutliche Präferenz der Patientinnen für die subkutane Gabe von Trastuzumab gegenüber der intravenösen nach. In der begleitenden Time-and-Motion-Studie reduzierten sich unter der subkutanen Therapie die effektive Behandlungszeit der Patientinnen und der insgesamt für die Verabreichung von Trastuzumab notwendige Zeitaufwand des medizinischen Personals. Erfahrungen aus 7 deutschen Zentren decken sich weitgehend mit den Studienergebnissen. Vonseiten der Patientinnen bestand auch in der Praxis eine klare Präferenz für die subkutane Anwendung von Trastuzumab, wobei die Zeitersparnis durch die subkutane Anwendung als größter Vorteil wahrgenommen wurde. Trotz geringerer Vergütung aufgrund der bestehenden Abrechnungsmodalitäten fiel die Bilanz der Zentren für die subkutane Applikationsform von Trastuzumab positiv aus. Für sie bestand der wichtigste Vorteil in einer größeren Terminflexibilität, die in einigen Zentren zu einer Verbesserung einer angespannten Situation bei dünner Personaldecke und in Belastungsspitzen beitrug. Konsens war allerdings auch, dass die Vergütung onkologischer Behandlungen unbedingt angepasst werden muss, um eine Deckung des realen Betreuungsaufwands auch bei geänderten Applikationsformen zu gewährleisten. 


\section{Introduction}

\section{$\nabla$}

Targeted anti-HER2 therapy with the monoclonal antibody trastuzumab (Herceptin ${ }^{\circledR}$ ) is now the standard therapy for patients with early and with metastatic HER2-positive breast cancer [15]. Patients with early-stage breast cancer receive trastuzumab as adjuvant therapy for a period of 1 year [4-6]. For patients with metastatic breast cancer, treatment with trastuzumab is usually continued at least to disease progression or even beyond (treatment beyond progression) [5].

Until recently, trastuzumab was administered intravenously only. Either in a three-weekly or in a weekly interval trastuzumab was adjusted to the patient's body weight. To allow a quick saturation of the HER2neu receptors treatment starts with a higher loading dose followed by a lower dose of trastuzumab to maintain the drug level [6].

Since August 2013 a more time-saving subcutaneous (s.c.) formulation of trastuzumab is available to treat patients with HER2-positive breast cancer. Subcutaneous trastuzumab (Herceptin ${ }^{\circledR} \mathrm{SC}$ ) is administered as a fixed dose of $600 \mathrm{mg}$ (irrespective of the patient's body weight) in a total fix volume of $5 \mathrm{ml}$. A loading dose is not required anymore. It is administered over a period of up to 5 minutes [7], while the time required for the intravenous infusion of trastuzumab is 90 minutes for the initial infusion and 30 minutes for each subsequent administration [6]. The follow-up times are the same for both forms of administration $[6,7]$.

This paper provides an overview of the study data on the subcutaneous administration of trastuzumab and summarizes and evaluates the experience of 7 German centers over 18 months of administering subcutaneous trastuzumab in routine clinical practice.

\section{Development of the Subcutaneous Formulation of Trastuzumab \\ $\nabla$}

The injection volume of the subcutaneous trastuzumab formulation is $5 \mathrm{ml}$. The injection of volumes greater than $2 \mathrm{ml}$ is usually painful and is limited by the physiology of the extracellular matrix of the subcutaneous tissue [8]. The co-formulation of trastuzumab with recombinant human hyaluronidase (rHuPH20) resulted in a temporary removal of this barrier (which consists of fibrous proteins, hyaluronan, glycosaminoglycans and other complex linear polysaccharides), allowing easy and painless, subcutaneous administration of trastuzumab [7-11].

To make administration as simple as possible and prevent dosing errors, a fixed-dose formulation, independent of the patient's body weight, was developed for subcutaneous administration of trastuzumab. With the fixed dose of $600 \mathrm{mg}$ sufficient concentration of the agent in serum as well as receptor saturation from cycle 1 are achieved [12]. The model was retrospectively validated using observed pharmacokinetic data from the randomized, phase III HannaH trial [12,13].

In the HannaH trial, 596 patients with HER2-positive, non-metastatic, locally advanced or inflammatory breast cancer received neoadjuvant chemotherapy. In addition, patients received trastuzumab either as a fixed dose of $600 \mathrm{mg}$ administered subcutaneously ( $\mathrm{n}=297$ ) or intravenously ( $8 \mathrm{mg} / \mathrm{kg}$ initial dose, $6 \mathrm{mg} / \mathrm{kg}$ maintenance dose) $(n=299)$ once every three weeks. Therapy with trastuzumab was continued after surgery until 18 cycles had been completed. HannaH was designed to show comparable pharmacokinetics for both administration forms of trastuzumab and an equivalent efficacy and tolerability $[14,15]$.

Drug serum concentration measured immediately pre-surgery, i.e., the geometric mean of the trough concentration $\left(\mathrm{C}_{\text {trough }}\right)$, was $69.0 \mu \mathrm{g} / \mathrm{ml}$ in the group receiving subcutaneous administration and $51.8 \mu \mathrm{g} / \mathrm{ml}$ in the group with intravenous (i.v.) administration. The mean ratio of the subcutaneous group to the intravenous group was 1.33 (90\% confidence interval [CI]: 1.24-1.44), well above the previously defined non-inferiority limit of 0.80 [14]. Pathological complete remission (pCR) was $45.4 \%$ for patients who received subcutaneous trastuzumab and $40.7 \%$ for patients who had intravenous trastuzumab [14].

The incidence of adverse events including events $\geq$ grade 3 was comparable for both study groups. Slightly more patients in the subcutaneous group experienced serious adverse events (SAEs) (21.5\%; 95\% CI: 17.0-26.7\%) compared to patients in the intravenous group (14.1\%; 95\% CI: $10.4-18.6 \%$ ). No association was found between toxicity and body weight or exposure [15].

A further analysis presented at ASCO 2015 showed that achieving tpCR (defined as the absence of invasive tumor cells in breast and axilla) resulted in a significantly better event-free survival independent of the respective application form of trastuzumab (comparison of tpCR vs. no tpCR: HR 0.38, 95\% CI 0.22-0.65 for s. c.; HR $0.32,95 \%$ CI $0.18-0.60$ for i.v.) [16]

The safety profile of subcutaneous trastuzumab is currently under evaluation in the SafeHer trial (ClinicalTrials.gov identifier: NCT01566721), a large, international, non-randomized, phase III trial of 2500 patients with early breast cancer divided into two cohorts to receive subcutaneous trastuzumab either in the form of assisted administration (conventional syringe and needle) or by self-administration (single-use injection device) [17].

\section{The PrefHer Trial - Experience of Patients and Healthcare Professionals \\ $\nabla$}

The randomized, two-cohort, crossover trial PrefHer systematically assessed patient preference for subcutaneous or intravenous administration of trastuzumab as well as the satisfaction of healthcare professionals with both treatment options. The study evaluated patient preference for subcutaneous administration using a conventional syringe or using a single-use injection device, and the time and resources required for the respective administration methods $[18,19]$.

Patients $(n=488$ ) were randomized into 2 groups after completion of surgery and chemotherapy (neoadjuvant and adjuvant). Patients randomized to study arm A $(n=245)$ received adjuvant trastuzumab therapy consisting of 4 cycles of trastuzumab administered subcutaneously (600 mg every 3 weeks), followed by 4 cycles of trastuzumab administered intravenously $(6 \mathrm{mg} / \mathrm{kg}$ every 3 weeks). The sequence was reversed for patients randomized to study arm B ( $\mathrm{n}=243)$, with patients in this arm initially receiving 4 cycles of trastuzumab administered intravenously (initial dose $8 \mathrm{mg} / \mathrm{kg}$, subsequently $6 \mathrm{mg} / \mathrm{kg}$ every three weeks from cycle 2), followed by 4 cycles of trastuzumab administered subcutaneously (600 mg every 3 weeks). Patients in cohort 1 received subcutaneous trastuzumab via a single-use injection device; patients in cohort 2 received trastuzumab via a hand-held syringe. After a crossover period of 8 cycles, trastuzumab was administered intravenously to patients in cohort 1 and subcutaneously by injection to patients in cohort 2 until the total therapy of 18 cycles had been completed ( $\bullet$ Fig. 1 ) [19]. 


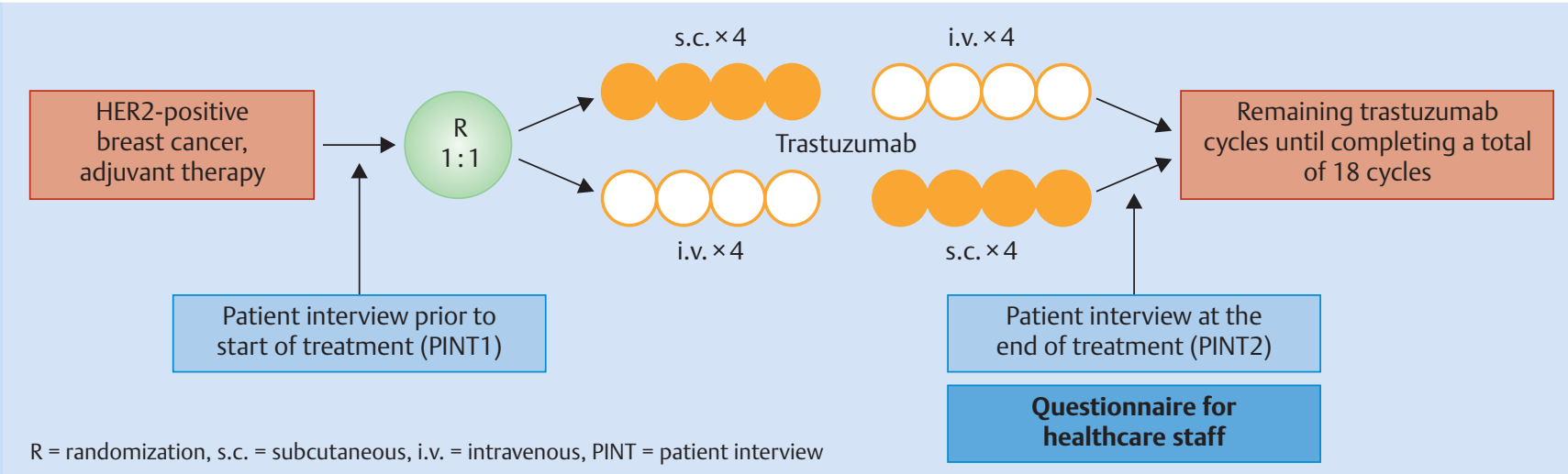

Fig. 1 Study design of the PrefHer study (based on $[18,19]$ ).

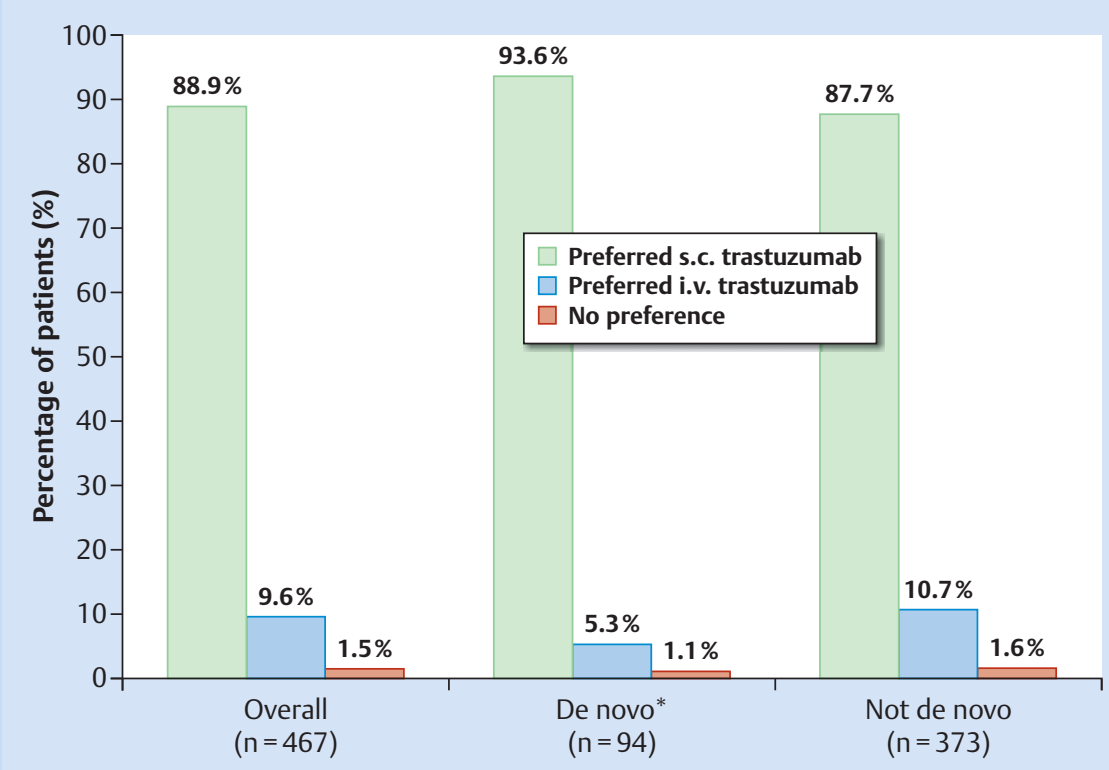

Fig. 2 Preference of patients for a specific type of trastuzumab administration (based on [19]).

* De novo: patients received no trastuzumab prior to inclusion in the study

The results revealed a significant preference of patients for subcutaneous trastuzumab administration, with $88.9 \%$ of patients preferring subcutaneous administration to intravenous administration (95\% CI: 85.7-91.6\%; p < 0.0001). 9.6\% of patients preferred intravenous administration and $1.5 \%$ had no preference ( $\bullet$ Fig. 2 ) [19].

The results were consistent for both study arms, i.e. irrespective of the order of trastuzumab administration (s.c. $\rightarrow$ i.v. or i.v. $\rightarrow$ s.c.). The preference for subcutaneous trastuzumab administration was reported as "very strong" by $64.9 \%$ of patients, "strong" by $17.3 \%$ of patients and as "not very strong" by $6.6 \%$ of patients. Both patients with a preference for subcutaneous administration existing prior to the start of therapy (203/216, 94.0\%) and patients who had previously indicated a preference for intravenous administration or no preference at all (212/251, 84.5\%) preferred subcutaneous administration after the crossover period. The most important reason for preferring subcutaneous trastuzumab administration cited by patients was the saving of time (mentioned by $80.3 \%$ ), followed by less pain (cited by $34.3 \%$ ) ( Table 1) [19].
Table 1 Primary reasons for patient preference (evaluable ITT population) (based on [19]).

\begin{tabular}{|c|c|}
\hline Reasons & n (\%) \\
\hline \multicolumn{2}{|c|}{$\begin{array}{l}\text { Preference for } \text { s. } c \text {. administration, } n=756 \text { reasons reported } \\
\text { by } 415 \text { patients }\end{array}$} \\
\hline Time saving & $375(80.3)$ \\
\hline Less pain/discomfort/side-effects & $160(34.3)$ \\
\hline Ease of administration & $62(13.3)$ \\
\hline Convenience to patients & $57(12.2)$ \\
\hline Problems with i.v. & $51(10.9)$ \\
\hline Less stress/anxiety & $35(7.5)$ \\
\hline Other & $20(4.3)$ \\
\hline \multicolumn{2}{|c|}{ Preference for $i$. $v$. administration, $n=64$ reasons reported by 45 patients } \\
\hline Fewer reactions (less pain, bruising, irritation, etc.) & $33(7.1)$ \\
\hline Other/don't know & $10(2.1)$ \\
\hline Psychological & $9(1.9)$ \\
\hline Perceived efficacy & $6(1.3)$ \\
\hline Environment/staff & $5(1.1)$ \\
\hline Ecological considerations & $1(0.2)$ \\
\hline
\end{tabular}

ITT: intention-to-treat; s. c.: subcutaneous; i. v.: intravenous. 


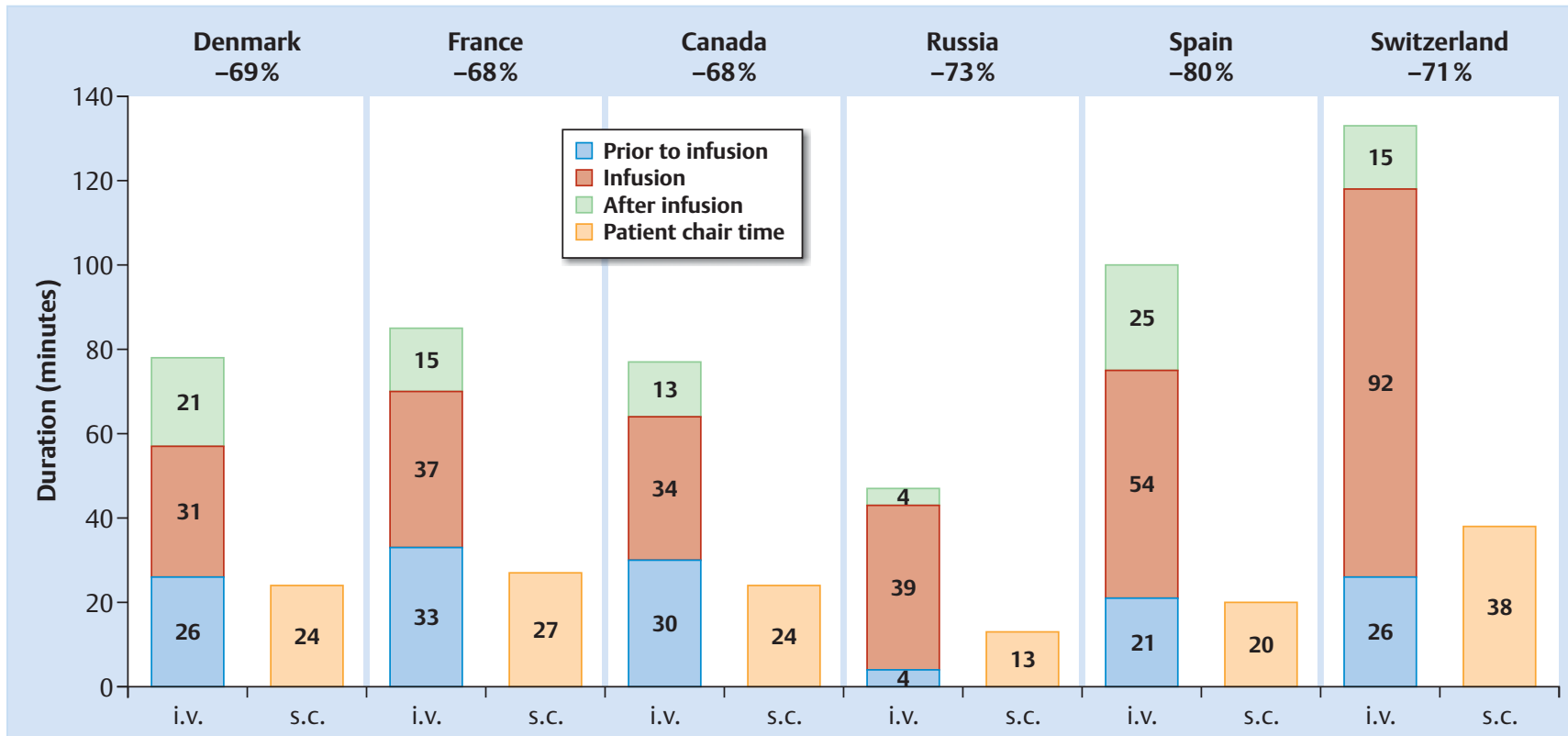

Fig. 3 Time spent by patient in the treatment chair (= patient chair time) - s. c. administration* vs. i.v. administration (based on [20]). * Subcutaneous administration of trastuzumab using single-use injection device.

Healthcare professionals also preferred subcutaneous administration, with 181 of 235 surveyed healthcare professionals reporting greater satisfaction with subcutaneous administration compared to intravenous administration (77\%, 95\% CI: 71.1$82.2 \%$ ). Seven of 235 healthcare professionals (3.0\%, 95\% CI: $1.2-$ $6.0 \%$ ) preferred intravenous administration, while 47 of 235 medical professionals (20\%, $95 \%$ CI: $15.1-25.7 \%$ ) had no preference for either method of administration [19].

\section{PrefHer Time-and-Motion Sub-study \\ $\nabla$}

A non-interventional prospective time-and-motion study was also performed as a sub-study of the PrefHer clinical study. This sub-study collected comparative data on the time spent by patients receiving trastuzumab (= patient "chair time") and the time spent by healthcare professionals administering trastuzumab in its different formulations. A total of 17 centers in Canada, Denmark, France, Russia, Spain and Switzerland participated in this sub-study $[19,20]$.

The results differed significantly between participating countries. However, in all centers the patient chair time was significantly lower for subcutaneous trastuzumab administration compared to intravenous trastuzumab administration. The absolute time saving per session ranged from 34 minutes (Russia) to 95 minutes (Switzerland), the relative time saving ranged from 68\% (Canada) to 80\% (Switzerland) (O Fig. 3). For treating 10 patients per year, time savings ranged from 103 hours (Russia) to 284 hours (Switzerland) for subcutaneous compared to intravenous administration of trastuzumab [20].

The active Healthcare Professional (HCP) time covered the total time spent by healthcare professionals on all tasks associated with the preparation and administration of trastuzumab. Here again, the time required to prepare and administer the subcutaneous trastuzumab formulation was significantly lower than for intravenous trastuzumab. The absolute time saving per session ranged from 7.4 minutes (Denmark) to 18.7 minutes (Canada), the relative time saving ranged from 30\% (Switzerland) to $51 \%$ (Spain). Projected to a treatment duration of 1 year consisting of 18 cycles, time savings ranged from 2.3 hours (Denmark) to 5.5 hours (Canada) per patient ( $\bullet$ Fig. 4) [20]. Patient chair times and active HCP times were lower for all forms of subcutaneous administration (i.e., single-use injection device and conventional injection via syringe), without significant differences between these administration forms [21].

In addition to quantitative data, the study also collected data on the qualitative benefits perceived for switching from intravenous to subcutaneous trastuzumab as assessed by HCPs. The data demonstrated that switching was associated with benefits for patients, nursing staff, physicians and the center itself, although it was felt that patients benefitted most [22].

\section{Experience of Administration of Subcutaneous Trastuzumab in the German Centers \\ $\nabla$}

The experiences of other healthcare systems may be of limited relevance for the situation in Germany since they are not necessarily transferable. German centers also participated in the PrefHer study but not in the time-and-motion sub-study. A project involving 7 German centers in which subcutaneous (s.c.) trastuzumab was administered regularly and successfully since it was first introduced aimed to provide a qualitative impression of trastuzumab s.c. administration in German clinical practice. The following centers participated in the project: Breast Cancer Center at Sana Klinikum Offenbach, Department of Gynecology, University of Hamburg-Eppendorf, Gynecological Hospital of the Municipal Hospitals Lüneburg, Department of Gynecology and Obstetrics at Hanover Medical School, Department of Gynecology and Obstetrics, University of Magdeburg, the Gynecological Hospital of the Municipal Hospitals Karlsruhe, and the Center for Outpatient Treatment in the Gynecologic Oncology Department 


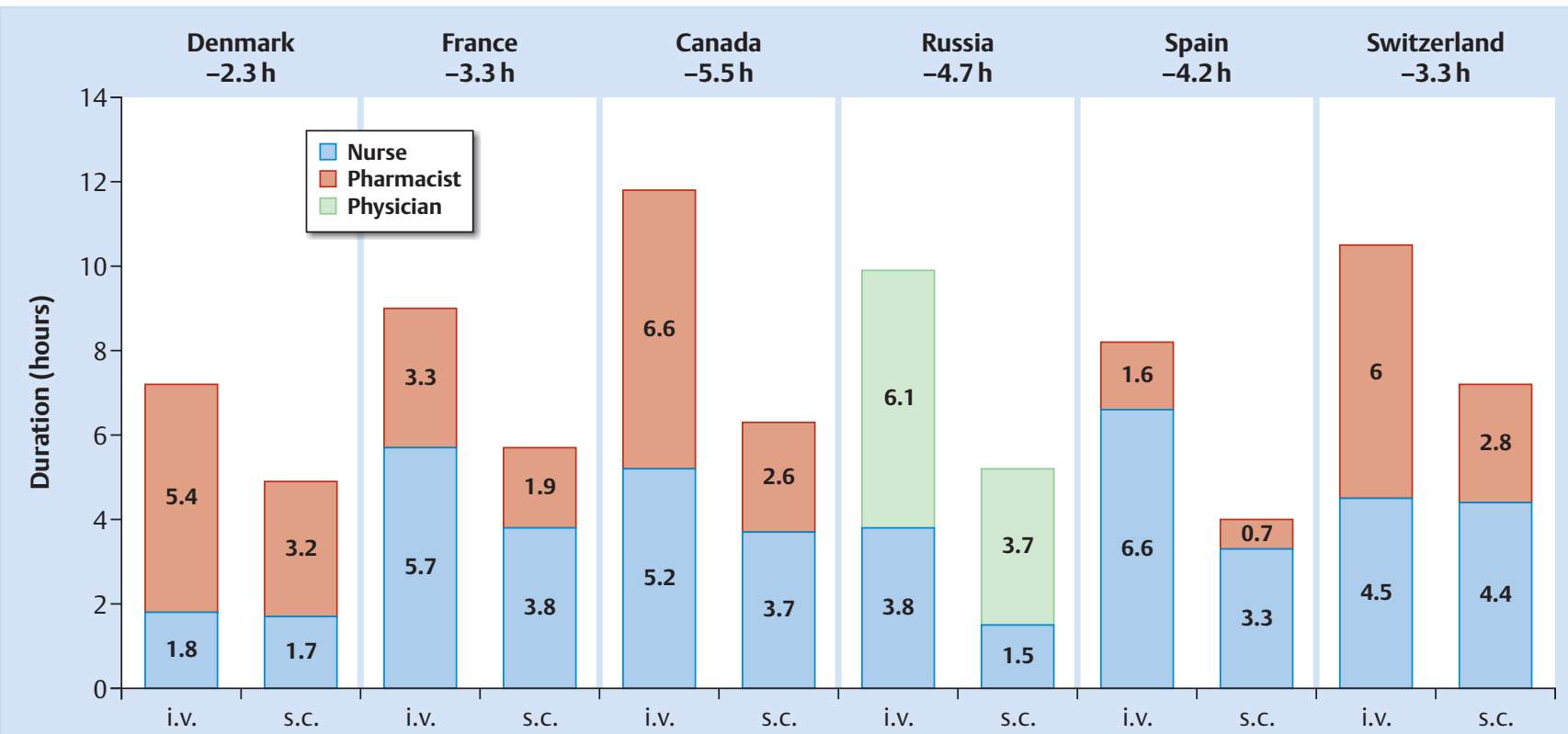

Fig. 4 Active Healthcare Professional (HCP) time required to administer trastuzumab to a patient over a period of 1 year - s.c. administration* vs. i.v. administration (based on [20]). ${ }^{*}$ Subcutaneous administration of trastuzumab using single-use injection device.

of the Hospitals Essen-Mitte. One physician responsible for oncologic outpatient care from every center was invited to attend a Metaplan ${ }^{\circledR}$-method workshop.

The rationale of the project was to describe experiences with trastuzumab s.c. outside clinical studies and to extrapolate best practices for trastuzumab s.c. administration. A structured telephone interview covering 6 related topics was conducted prior to the workshop with the attending physician from each center. The results of these qualitative interviews were summarized, presented during the moderated workshop, and adjusted based on the questions raised by the participants during the workshop.

\section{Why the Centers Introduced Subcutaneous Trastuzumab}

There were multiple reasons to prompt centers to introduce subcutaneous trastuzumab. The expected benefit for patients was an important consideration for all of the centers.

Even before trastuzumab s.c. was approved for routine clinical use, 4 centers already had some prior practical experience of administering subcutaneous trastuzumab through participation in clinical studies. For 2 other centers, the results of the HannaH and the PrefHer studies were the decisive factors motivating them to introduce trastuzumab s.c. in clinical practice.

In all of the centers, the switch to trastuzumab s.c. was done in close cooperation with the respective pharmacy services. In one case, the initiative to switch to trastuzumab s.c. came directly from the pharmacist himself, who considered it as an opportunity to free up additional capacity and relieve the strain on the department responsible for preparing cytostatic drugs. Although not the primary reason, capacity bottlenecks also played a role in the decision of several centers to introduce trastuzumab s.c.. In one case, the switch was linked to the expectation of reducing the amount of overtime of the nursing staff. In 2 cases, the num- ber of chairs available for intravenous therapy was insufficient at peak times and switching was hoped to ease this pressure.

Most centers hoped that switching to trastuzumab s.c. would result in more flexibility in scheduling appointments.

\section{Structure of the Centers \\ $\nabla$}

The healthcare settings in which patients received subcutaneous trastuzumab varied; they included a university hospital outpatient department $(\mathrm{n}=1)$, a university hospital outpatient department with day-patient billing $(n=1)$, outpatient departments as defined in $\S 116 \mathrm{~b}$ of the German Social Welfare Code, Book V $(n=3)$, an outpatient clinic as defined in $\S 116 \mathrm{~b}$ of the German Social Welfare Code, Book V in cooperation with an oncologist in private practice with shared consulting rooms on the hospital grounds $(n=1)$, in hospital and in cooperation with an oncologist in private practice with consulting rooms on the hospital grounds $(\mathrm{n}=1)$.

The number of patients treated ranged from 100-600 patients with early breast cancer and 20-180 patients with metastatic breast cancer per year per center.

\section{Logistics and Preparation}

Appointments to receive subcutaneous trastuzumab were usually made with more flexibility than appointments for intravenous administration and could therefore also occasionally be "squeezed in". Some centers strictly switched to afternoon appointments for patients receiving subcutaneous trastuzumab.

The centers usually ordered subcutaneous trastuzumab preparations for individual patients the day before therapy. In one center, the order was prepared in advance but only completed on the day of treatment when the patient presented at the center. In another center, the order was placed on the scheduled treatment day if 
the patient had not cancelled her appointment by 10:00 a.m. In another center the order was placed three days prior to the start of therapy.

Trastuzumab preparations were usually supplied by the center's in-house pharmacy. Subcutaneous trastuzumab was supplied either as a prefilled syringe or in a vial. One center switched from vials to prefilled syringes after a few months, while another center switched from syringes filled by the pharmacy to vials. In each case, the change was made for specific local organizational reasons after consultation with the supplying pharmacy. When subcutaneous trastuzumab is supplied as a vial, the medical staff of the center fills the syringe immediately prior to administration. These centers keep a supply of 3-5 vials of subcutaneous trastuzumab in reserve.

The recommended dose for subcutaneous trastuzumab is $600 \mathrm{mg}$, irrespective of the patient's body weight. The charged syringe has a shelf life of 48 hours when kept at a temperature of $2-8{ }^{\circ} \mathrm{C}$ and a further 6 hours at room temperature (up to $30^{\circ} \mathrm{C}$ ) in diffuse daylight [7]; because of the fixed dose, prefilled syringes kept at the recommended temperatures could, in principle, also be used for treating another patient within this period. This means that no wastage occurs in centers treating sufficient numbers of patients, irrespective of supply as prefilled syringe or vial.

\section{Administration}

\section{$\nabla$}

The trastuzumab formulation is administered subcutaneously every three weeks; administration is done in 2-5 minutes. In some centers, the injection is administered by nursing staff, in a few centers it is administered by a physician. In two centers subcutaneous trastuzumab is administered either by a physician or by nursing staff, depending on availability. None of the centers assigned a special room for administration. The single-use injection device was not used in any of the centers as the device is not yet available for general clinical use.

\section{Which Patients Received Subcutaneous Trastuzumab? $\nabla$}

In principle, with the exception of patients enrolled in clinical trials, the centers offered every patient for whom trastuzumab therapy was indicated, a choice between the two forms of administration. Ultimately it was the patient who decided whether she wanted to receive intravenous or subcutaneous trastuzumab. There were only a few exceptions to this rule. Three centers preferred weekly intravenous administrations of trastuzumab in selected high-risk patients with cardiovascular risk factors. This ensured that these patients were closely monitored, with the expectation that intervention would be quicker in the event of any cardiac side-effects. In one center patients with severe dermatologic diseases such as psoriasis were advised against subcutaneous therapy.

All surveyed centers preferred subcutaneous trastuzumab if no other drug therapy was administered in parallel.

Patients receiving chemotherapy or another intravenous antibody therapy in addition usually received intravenous trastuzumab, particularly if a central venous port was already in place, even though subcutaneous administration would have also significantly reduced the time spent in the center by these patients. In these cases, avoiding additional injections was given prefer- ence over potential savings of time. Only one of the centers administered subcutaneous trastuzumab during the chemotherapy phase if the patient requested it.

The centers used different approaches for switching patients who were already receiving ongoing trastuzumab therapy. Three centers aimed to switch all patients receiving trastuzumab monotherapy to subcutaneous trastuzumab. A few patients refused to switch. One center agreed on a trial period with its patients after which they would have the opportunity to revert to intravenous trastuzumab. However all patients wanted to continue with subcutaneous administration. The other centers continued intravenous administration in patients who were already undergoing trastuzumab therapy. Patients were only switched over if the patient herself requested the change.

\section{Experience of the Centers after More Than One Year of Subcutaneous Trastuzumab Use $\nabla$}

Patient acceptance of subcutaneous trastuzumab in routine care was very high in the 7 centers. The majority of patients (70-90\%) opted for subcutaneous trastuzumab after being offered both administration forms. Their feedback was almost entirely positive and compliance was very good. The time saving issue was cited as the most important benefit. In contrast to orally administered therapies patients who received subcutaneous trastuzumab felt well taken care of. They valued the increased flexibility in making appointments which made it easier to integrate therapy into daily life, for example by receiving trastuzumab treatment in the morning before starting work. At the same time, this experience gave rise to the expectation that procedures could be done even faster; for example, that treatment could be administered without having to wait for a consultation with a doctor. The centers also noted a perceptible increase in the numbers of patients actively requesting subcutaneous trastuzumab, possibly due to an exchange of information and of experiences in the center and on internet forums.

The experiences reported by the healthcare professionals of the centers in qualitative interviews were also positive. Most found administering trastuzumab subcutaneously to be simpler, although the barrier to administering a subcutaneous injection is clearly lower than for placing a venous access device. In the experience of the centers, the actual cumulative workload and the time spent by healthcare staff on all activities related to trastuzumab administration did not differ significantly between subcutaneous and intravenous administration. Because subcutaneous trastuzumab administration allows appointments to be made more flexibly, switching from intravenous to subcutaneous administration simplified everyday routines in the centers, freeing up capacity for intravenous therapies. This flexibility helped prevent overhours and kept staffing costs stable. It helped relieve situations when staffing levels were low. However, because of current accounting rules and the lower reimbursement of subcutaneous therapy, switching to subcutaneous trastuzumab can result in a real loss in income compared to treatment with intravenous trastuzumab.

The current accounting and reimbursement system does not adequately mirror the benefits of subcutaneous injection compared to intravenous administration. The absolute financial loss per patient in real terms depends on the currently applying accounting guidelines which, in turn, depend on the respective local Association of Statutory Health Insurance Physicians (Kassenärztliche 
Vereinigung [KV]). In the Physicians' Fee Schedule (einheitlicher Bewertungsmaßstab [EBM]), the reimbursement for the intravenous administration of trastuzumab per infusion day according to EBM code 02101 amounts to $€ 16.13$. If the center is entitled to an additional reimbursement under the terms of the Oncology Agreement (Onkologie-Vereinbarung [OV]), OV code 86516 allows the center to invoice an additional sum of between $€ 141.27$ and $€ 255.65$ (depending on the respective health insurance association) per quarter per patient treated with intravenous trastuzumab. If a patient receives subcutaneous trastuzumab, both EBM code 02101 and OV code 8651 do not apply.

In terms of reimbursement systems, the federal states of Saxony and Thuringia are models other states would do well to emulate. The two states introduced a separate code for the reimbursement of oral oncologic therapies several years ago. A supplement to this code for oral treatment including reimbursement of subcutaneous oncologic therapy was introduced recently in Saxony and is under discussion in Thuringia.

\section{Discussion}

$\nabla$

The experiences of the 7 centers with the administration of subcutaneous trastuzumab correspond in many aspects with the results of the PrefHer trial. Patients had a clear preference for subcutaneous administration of trastuzumab. Most patients had previously received intravenous trastuzumab administered in parallel to chemotherapy and were therefore able to compare both forms of administration. As demonstrated in the time-andmotion study, subcutaneous trastuzumab resulted in a relevant time saving for patients. It needs to be pointed out that the survey of the centers did not permit quantitative conclusions but only qualitative statements. The time saving was perceived by patients as a significant benefit. They also appreciated the greater flexibility in making appointments allowing them to live a more flexible life while undergoing systemic long-term oncologic therapy.

The overall assessment of the centers after using subcutaneous trastuzumab for more than one year was positive ( $\bullet$ Table 2 ). Their expectations of greater flexibility were realized. The clear benefit for patients and the higher levels of patient satisfaction were the most important findings. Subcutaneous trastuzumab offered the centers more room for maneuver as it allowed them to be more flexible when scheduling appointments even though staffing levels remained low. This is of major importance because various factors, including the increased complexity of oncologic therapies, the challenges posed by the lack of staff, and stricter compliance with working time regulations, has made providing outpatient oncologic therapies more difficult for centers. For the centers, safety aspects were an additional important reason supporting the switch to subcutaneous trastuzumab. The fixed dose reduces the likelihood of dosing errors. Moreover, subcutaneous trastuzumab therapy always uses an original active pharmaceutical agent (API) with a known efficacy and tolerability profile. No differences between intravenous trastuzumab and subcutaneous trastuzumab with regard to efficacy and tolerability were found in clinical practice; there was also no increase in the incidence of SAEs. However, a word of caution is necessary: these assessments were based on subjective observations and not on a systematic analysis of patient data. But, ultimately, each of the 7 centers would opt for the same decision today to switch to subcutaneous trastuzumab.
Table 2 Overall assessment of benefits and practical challenges associated with switching from intravenous to subcutaneous or oral administration.

\begin{tabular}{|c|c|}
\hline For & Against \\
\hline \multicolumn{2}{|l|}{ Patient } \\
\hline $\begin{array}{l}\text { Saving of time } \\
\text { Greater flexibility in obtaining appointments } \\
\text { Therapy can be integrated more easily in daily } \\
\text { activities } \\
\text { Original active pharmaceutical ingredient with } \\
\text { verified efficacy and tolerability profile } \\
\text { Prevents dosing errors } \\
\text { Equivalent efficacy and tolerability of subcuta- } \\
\text { neous and intravenous trastuzumab }\end{array}$ & $\begin{array}{l}\text { Better control with } \\
\text { intravenous tras- } \\
\text { tuzumab when } \\
\text { administering } \\
\text { therapy to cardiac } \\
\text { high-risk patients }\end{array}$ \\
\hline \multicolumn{2}{|l|}{ Center } \\
\hline $\begin{array}{l}\text { Prevents dosing errors } \\
\text { Prevents wastage } \\
\text { - Greater flexibility in making appointments } \\
\text { - Relieves pressures on the clinic in peak times } \\
\text { Prevents overtime } \\
\text { - Compensates for a lack of staff } \\
\text { Frees up capacity for intravenous therapies } \\
\text { Competitive advantage over other centers } \\
\text { which do not offer patients the benefit and the } \\
\text { saving of time associated with subcutaneous } \\
\text { trastuzumab administration }\end{array}$ & $\begin{array}{l}\text { Overall working } \\
\text { hours and time } \\
\text { required identical, } \\
\text { irrespective of the } \\
\text { form of administra- } \\
\text { tion } \\
\text { Reimbursement of } \\
\text { subcutaneous tras- } \\
\text { tuzumab is lower }\end{array}$ \\
\hline
\end{tabular}

After all, all centers see that a fairer reimbursement system is urgently needed. More and more modern oncologic therapies are not being administered intravenously but orally or subcutaneously. The actual administration may, indeed, require less time. However, subcutaneous or oral therapies still require healthcare professionals and medical staff to provide time-consuming services, including extensive consultations with patients and monitoring of side-effects, none of which are adequately covered by the current reimbursement systems. It is obviously a problem if, for economic reasons, patients are not offered new therapeutic options which offer a better quality of life because - as in the case of trastuzumab - the reimbursement for conventional intravenous administration is higher. A center offering this kind of therapy that is more comfortable for patients should not experience financial problems only because the current reimbursement policy does not adequately reimburse these services. According to figures from December 2014, subcutaneous trastuzumab administration accounted for a mere $14.0 \%$ of the total amount of trastuzumab administered in Germany. The percentage for the same period was $23.7 \%$ in France, $60.1 \%$ in the United Kingdom and as high as $76.4 \%$ in Sweden [23]. Oncological healthcare systems and the reimbursement systems in the latter countries are different from those in Germany. There are probably a number of reasons for this disparity in the acceptance of new application modalities for trastuzumab, despite the proven efficacy and patient preference for this new option. But these figures also demonstrate that patient access to modern oncologic therapies is significantly affected by existing reimbursement systems.

\section{Conclusion \\ $\nabla$}

In view of the recent advances and expected progress in oncologic therapies, a systematic review of the current reimbursement systems for outpatient care and adequate reimbursement options for modern outpatient oncologic therapy are urgently required. 


\section{Conflict of Interest}

\section{$\nabla$}

The authors declare that in the last 3 years they have provided the following services or received the following support or grants: CJ speaker's fees and travel expenses from Roche; VM consultancy fees and speaker's fees from Amgen, Celgene, Eisai, Pierre Fabre, Roche as well as research funding from Celgene and Roche; PD speaker's fees and travel expenses from Roche; RN speaker's fees from Roche; T-WP-S consultancy fees and speaker's fees from Roche; SS consultancy fees and speaker's fees from Astra-Zeneca, GSK, Novartis, Riemser, Roche, TEVA; HT consultancy fees and speaker's fees from Roche; BA consultancy fees and speaker's fees from Roche.

\section{Affiliations}

${ }^{1}$ Klinik für Gynäkologie und Geburtshilfe, Sana Klinikum Offenbach, Offenbach

2 Frauenklinik, Universitätsklinikum Hamburg-Eppendorf, Hamburg

${ }^{3}$ Frauenklinik, Städtisches Klinikum Lüneburg, Lüneburg

${ }^{4}$ Frauenklinik, Universitätsklinikum Magdeburg, Magdeburg

${ }^{5}$ Klinik für Frauenheilkunde und Geburtshilfe, Medizinische Hochschule Hannover, Hannover

${ }^{6}$ Frauenklinik, Städtisches Klinikum Karlsruhe, Karlsruhe

7 Onkologische Gemeinschaftspraxis am Bethanien-Krankenhaus, Frankfurt

${ }^{8}$ Gynäkologie und Gynäkologische Onkologie, Kliniken Essen-Mitte, Essen

\section{Literatur}

1 Giordano SH, Temin S, Kirshner JJ et al. Systemic therapy for patients with advanced human epidermal growth factor receptor 2-positive breast cancer: American Society of Clinical Oncology clinical practice guideline. J Clin Oncol 2014; 32: 2078-2099

2 Senkus E, Kyriakides S, Penault-Llorca F et al.; ESMO Guidelines Working Group Primary breast cancer. ESMO Clinical Practice Guidelines for diagnosis, treatment and follow-up. Ann Oncol 2013; 24 (Suppl. 6): vi7vi23

3 Cardoso F, Costa A, Norton L et al. ESO-ESMO 2nd international consensus guidelines for advanced breast cancer (ABC2)†. Ann Oncol 2014; 25: $1871-1888$

4 DKG. Interdisziplinäre S3-Leitlinie für die Diagnostik, Therapie und Nachsorge des Mammakarzinoms Langversion 3.0, Aktualisierung 2012. Online: http://www.krebsgesellschaft.de/download/S3_Brustkrebs_Update_2012_OL_Langversion.pdf; last access: 03.03.2015

5 AGO. Diagnostik und Therapie von Patientinnen mit primärem und metastasiertem Brustkrebs: Version 2015. 1D [online] 03.2015. Online: http://www.ago-online.de/fileadmin/downloads/leitlinien/ mamma/maerz2015/de/2015D_Alle_aktuellen_Empfehlungen.pdf; last access: 03.03.2015

6 Aktuelle Fachinformation Herceptin ${ }^{\circledR}$, April 2015

7 Aktuelle Fachinformation Herceptin ${ }^{\circledR}$ SC, April 2015

8 Haller F. Converting intravenous dosing to subcutaneous dosing with recombinant human hyaluronidase. Pharm Tech 2007; 10: 861-864
9 Watson D. Hyaluronidase. Br J Anaesth 1993; 71: 422-425

10 Bookbinder LH, Hofer A, Haller MF et al. A recombinant human enzyme for enhanced interstitial transport of therapeutics. J Control Release 2006; 114: 230-241

11 Frost GI. Recombinant human hyaluronidase (rHuPH20): an enabling platform for subcutaneous drug and fluid administration. Expert Opin Drug Deliv 2007; 4: 427-440

12 Hourcade-Potelleret F, Lemenuel-Diot A, McIntyre C et al. Use of a population pharmacokinetic approach for the clinical development of a fixed-dose subcutaneous formulation of trastuzumab. CPT Pharmacometrics Syst Pharmacol 2014; 3: e87

13 Wynne C, Harvey V, Schwabe C et al. Comparison of subcutaneous and intravenous administration of trastuzumab: a phase $\mathrm{I} / \mathrm{Ib}$ trial in healthy male volunteers and patients with HER2-positive breast cancer. J Clin Pharmacol 2013; 53: 192-201

14 Ismael G, Hegg R, Muehlbauer S et al. Subcutaneous versus intravenous administration of (neo)adjuvant trastuzumab in patients with HER2positive, clinical stage I-III breast cancer (HannaH study): a phase 3, open-label, multicentre, randomised trial. Lancet Oncol 2012; 13: 869-878

15 Jackisch C, Kim S, Semiglazov V et al. Subcutaneous versus intravenous formulation of trastuzumab for HER2-positive early breast cancer: updated results from the phase III HannaH study. Ann Oncol 2015; 26: 320-325

16 Jackisch C, Hegg R, Stroyakovskiy D et al. Total pathologic complete response (tpCR) and event-free survival (EFS) with subcutaneous (SC) or intravenous (IV) trastuzumab in HER2-positive early breast cancer (EBC): The HannaH phase III study. J Clin Oncol 2015; 33 (Suppl.): Abstr. 585

17 Clinicaltrials.gov. Online: https://clinicaltrials.gov/ct2/show/NCT0156 6721?term=safeher\&rank $=1$; last access: 03.03 .2015

18 Pivot X, Gligorov J, Müller Vet al. Preference for subcutaneous or intravenous administration of trastuzumab in patients with HER2-positive early breast cancer (PrefHer): an open-label randomised study. Lancet Oncol 2013; 14: 962-970

19 Pivot X, Gligorov J, Müller V et al. Patients' preferences for subcutaneous trastuzumab versus conventional intravenous infusion for the adjuvant treatment of HER2-positive early breast cancer: final analysis of 488 patients in the international, randomized, two-cohort PrefHer study. Ann Oncol 2014; 25: 1979-1987

20 De Cock E, Semiglazov V, Lopez-Vivanco G et al. Time savings with trastuzumab subcutaneous vs. intravenous administration: a time and motion study. St. Gallen 2013, Abstr. P209

21 De Cock E, Knoop A, Jacobsen EH et al. Manual injection of subcutaneous trastuzumab vs. intravenous infusion for HER2-positive early breast cancer: a time-and-motion study. European Cancer Congress 2013, Amsterdam, Abstr. 1965

22 De Cock E, Pan I, Sandoval M et al. Healthcare professionals' perceptions of the impact on clinical management of switching from the intravenous to the subcutaneous formulation of trastuzumab. European Breast Cancer Conference 2014, Glasgow, Abstr. 42

23 Roche Data on file 